\title{
DESIGNING EMAIL MARKETING CAMPAIGNS - A DATA MINING APPROACH BASED ON CONSUMER PREFERENCES
}

\author{
Radu Ioan Mogoș ${ }^{1}$ \\ Carmen Acatrine ${ }^{2}$
}

\begin{abstract}
The organizations should approach the consumers by using only the instruments accepted and trusted by them and if the email is one of the instruments, companies must be able to design email marketing communication campaigns that would have a high response rate (either in what concerns the open rate, click rate and / or conversion rate). By owning a database and by designing email marketing campaigns, the organizations can send individually, personalized offers. The messages used for writing the emails should be adapted to the requirements of the target, based on some common characteristics and / or behaviors. These common characteristics can be found out by analyzing the interaction that the potential client has with the company's website, through personal designation of interests and preferences, by analyzing the buying behavior of the products visualized and the time spent on the website before / after the purchase was made. The present paper presents the results of a research among the factors that influence the recipients to open direct emails and make an action desired by the company and also studies whether and what elements in the email would influence them to buy the products / services promoted. The results are obtained based on a data mining analysis which includes clustering and classification processes and offer a guide on how organizations should design their email marketing communications in order to have higher response rates.
\end{abstract}

Keywords: email marketing campaigns, email customization, data mining analysis, consumer preferences

JEL codes: M31, M37, C38

\section{INTRODUCTION}

The internet makes a change of focus: from the product, towards the client, offering in this way multiple possibilities for personalization, both of the messages, as well as the offers and direct communication between the company / organization / brand and the consumer or potential buyer. All these are meant in order to satisfy the needs of the prospect with offers that are meant to convince him to buy, or to buy again the products / services offered by the company. Hence, the organizations need to know how to settle primary grounds of the internal processes in order to generate positive effects on the long term (Cioană, Dinulescu, 2015, p. 3096). Possible positive effects wanted by the organizations could also include a balance between the demands of the various stakeholders the organization has (Hurduzeu, 2015, p. 289) and this balance can only be attained if the proper tools are employed. Online communication tools can easily be used by organizations in order to transmit the messages wanted to the community, customers, employees or other stakeholders.

Within organizations, the terms "newsletter" and "email marketing campaign" are used as

\footnotetext{
${ }^{1}$ Bucharest University of Economic Studies, Faculty of Economic Cybernetics, Statistics and Informatics, 15-17 Calea Dorobanți, sector 1, 010552, Bucharest, Romania, mogos.radu@gmail.com

2 Bucharest University of Economic Studies, Faculty of Marketing, 41 Dacia Blvd., sector 1, 010404, Bucharest, Romania, carmen.acatrinei@mk.ase.ro
} 
synonyms, but the literature makes a clear distinction between the two concepts (Pantea and Pop, 2010, p. 739), highlighting the different scope of each tool. Email marketing campaigns are designed in order to generate a reaction on behalf of the recipient, a direct response; whereas the newsletter's purpose is to inform the reader, also to deliver good quality content; moreover the layout / format of the two emails are different and sometimes the companies not necessary include a personalized greeting formula. Newsletters can be used in order to maintain and develop long term relationships with stakeholders. Some of the similarities, between the two instruments, consist in the use of the email as the channel by which the message is transmitted, the existence of a database with customers and the need to have the consent of the recipients in order to send the email and both tools include one or two hyperlinks to the website of the organization.

Email marketing campaigns represent one of the most popular and efficient online marketing instruments. Building email marketing campaigns can help the organizations keep the current customers, motivate them not to abandon the shopping basket, it may convince the targeted public to make an acquisition, by making for instance a personalized offer, based on that person's needs and wants. Sending personalized offers has become a differentiating element, but in the same time, a barrier in the competition to attract new clients or to keep and make the existing clients loyal to the promoted brand (Friedlein, 2003, p. 241).

The paper is structured into four parts. The first part makes an analysis of the literature review on the topic of email marketing campaigns, the second one describes the methodology used in the exploratory research and the third part describes the results obtained using data mining techniques. The paper ends with conclusions, limitations and possible future directions of research.

\section{LITERATURE REVIEW}

Personalization of the messages and offers is a characteristic of the email marketing campaigns, together with the person's agreement to use his / her email address for sending commercial messages. In case the person is exposed to email marketing campaign without his / her consent, or if the message / offer transmitted is not relevant for the needs of the persons in the database, the company is actually spamming the consumer (Grimes et al., 2007, p. 319). Blyth (2011, p. 76) also considers that sending irrelevant emails to prospects is the new spam (similar to sending marketing messages to a random group of people) and should not be done by organizations.

In the process of designing email marketing campaigns, there are suggested some stages: defining the objectives of the campaign, determining target recipients, develop content, develop response, test, send, respond and finally, measurement of the results obtained (Gay et al., 2007, p. 406). The present paper focuses on researching deeply the content developing stage, by studying the elements needed to be taken into consideration when designing a direct emailing campaigns.

Most of the authors highlight the importance of the subject of the email (Blyth, 2011, p. 76; Charlesworth, 2009, p. 260) and its shortness so that it can be better displayed on mobile devices (Fallon, 2014), Vegheș (2003, p. 351) resembles it with a door knock (the email is seen, if the subject draws attention, the message will be opened, if not, it will probably be deleted). Other authors emphasize the importance of aspects such as: being brief and relevant for the target, including a personalized greeting at the beginning, including a hyperlink to website for more detailed content, clear call-to-action within the message, provide unsubscribing options (Chaffey, Smith, 2008, p. 328). Gay et al. (2007, p. 407) also stress upon the right subject line, the personal greetings, the call-to-action, in addition, it is drawn attention over the email address / domain name that needs to be recognized by the recipient, keeping the content concise and inclusion of a link (which is visible), the importance of the first paragraph that should contain important information. Other sources include aspects such as: inclusion of the company logo, inclusion of an image to make the message more appealing, benefit not feature driven content (Yola, 2013), inclusion of an alt text for the images so that their name is displayed even if the recipient does not view in HTML 
the picture (Shah, 2013); make valuable offers to give the recipients motivation to open emails (Ford, 2014); include social-sharing features: forward to a friend, buttons for sharing on Facebook, Twitter, Google+ (Handley, 2012); include a PS in order to remind the recipients of a deadline or to repeat what they can lose if they do not make an action towards obtaining the offer (Duistermaat, 2013). The email cannot be used as an instrument to sell, because the recipient will not be able to make a purchase directly from his / her email address, reason for which it is very important the landing page where the reader is driven after clicking the hyperlink/s present in the email. The landing page should be relevant for the message transmitted so that it has better chances to convert (Usadata.com)

In order to deliver the emails to the database owned, an organization can choose the services offered by different providers, such as: MailChimp, AWeber, Mad Mimi, CakeMail, Vertical Response, Benchmark, Constant Contact, iContact, ActiveCampaign, GetResponse, StreamSend (Angeles, 2014), SendinBlue and White Image (from Romania).

\section{METHDOLOGICAL NOTES}

Several aspects, related to the factors that influence a recipient to open a commercial email and to buy the product / service promoted in the email (or do another action wanted by the sender: attend an event, forward the email to friends etc.), have been assessed through this exploratory research approach. The data has been gathered at the level of sample including 262 respondents (91.23\% from urban environment, 8.77\% from rural environment), aged 20 to 35, with a higher education, and being either employees, freelancers, entrepreneurs, managers, and / or students. The data has been collected between April - May 2015 through an online survey, by using the snowball sampling method.

The research directions include: $(\mathrm{O} 1)$ determining the most important factors that influence the customers to have a positive reaction to an email received from a company that promotes a product / service; (O2) determining the most important factors that influence the customer to buy a product/service promoted by email by a company; (S1) identifying clients that belong to a group that have similar expectations (for $\mathrm{O} 1$ and $\mathrm{O} 2$ ); and (S2) identifying customers' profiles that make up the groups (clusters) (for O1 and O2).

Data mining dataset comprises 262 records and 22 attributes, all attributes being nominal. The main preprocess activities done for the data mining process were: the fill-out level of the data mining dataset was checked and fulfilled with null values were it was necessary; an ARFF file (Attribute Relation File Format) was created based on the dataset; there were selected the main analysis directions for the research; for each direction there were identified the relevance of each attribute, the clusters (groups of records) that have similar properties, decision rules for the identified profiles.

The data mining methodology used was Cross Industry Standard Process for Data Mining CRISP - DM and the methodological steps were followed: business understanding, data understanding, data preparation, modeling, evaluation, deployment (Chapman et. al, 2000). In order to analyze the dataset and other algorithms ("GainRatioAttributeEval" attribute evaluator and "Ranker" ordering method for selecting attributes; "SimpleKMeans" clustering algorithm for grouping similar clients in groups; and "Part" decision list algorithm to generate rules for different clients profiles), the authors used WEKA data mining software (Waikato, 2015). The steps done in order to obtain the final results were: creating the ARFF file in order to be opened in WEKA software; filtering and selecting attributes for the main analysis directions; computing a rank for each attribute related to the chosen class attribute; creating a number of clusters for clients with similar expectations (SimpleKMeans); clients' profiles identification based on a classification process (decision rules).

In order to identify the email aspects, the respondents were asked what determines them to 
open in email and how what does it influence them to respond with a positive action to the company's message. In Table 1 there is presented a description of these aspects.

Table 1.

Email aspects researched

\begin{tabular}{|l|l|l|}
\hline Aspect & Attribute \\
\hline $\begin{array}{l}\text { What determines } \\
\text { the client to open }\end{array}$ & - & Convincing subject \\
the email (Q20) & - & The organization name appearance \\
& - & Other reasons \\
\hline $\begin{array}{l}\text { What determines } \\
\text { the client to }\end{array}$ & - & A company logo has to be included within the email \\
respond with a & - & A personalized greeting to be comprised within the email \\
positive action to & - & A representative picture to be comprised within the email \\
the email & - & A funny message to be comprised within the email \\
according to the & - & Enough details to be comprised in the email, without being necessary \\
company & - & an additional link in the email \\
expectations? & - & The email to have relevant information for the client \\
(Q21) & - & A personalized ending greeting to be included \\
& - & The name of the employer who signs the email to be included \\
& - & A clear message \\
& - & A concise message \\
& - & A promotional offer to be included within the email \\
& - & It must not be intrusive \\
Email influence & - & The email must to be convincing \\
(Q4, Q23) & - & To buy the promoted product / service \\
& - & To react according to the company expectations when receiving a \\
&
\end{tabular}

The WEKA software offers the possibility to analyze the attributes (Fig. 1) and to obtain information about them. 


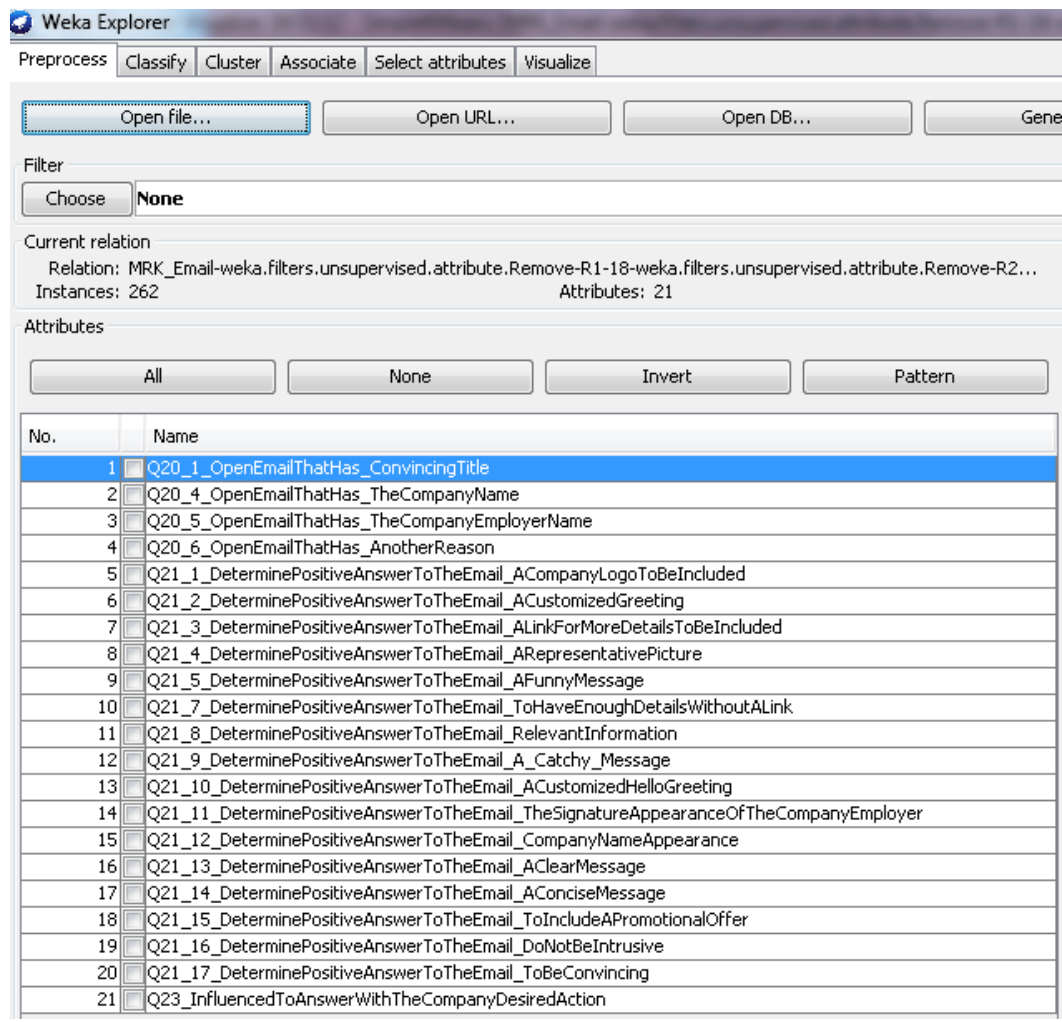

\section{MAIN FINDINGS}

Fig.1 The selected attributes in WEKA software

In order to follow the research directions settled, the data mining analysis has comprised an attribute selection and ranking according to a class attribute (A), a clustering process (B) and a classification process $(C)$.

In order to identify the most important factors that influence the customers to have a positive reaction to an email received from a company that promotes a product / service $(\boldsymbol{O I})$ it was selected the class attribute $Q 23$ - InfluencedToAnswerWithTheCompanyDesiredAction.

A. Ranking attributes: this step was done in order to identify the relation between the class attribute and the others. Using "GainRatioAttributeEval" attribute evaluator and "Ranker" ordering method for selecting attributes, there were obtained the following results:

Attribute Evaluator (supervised, Class (nominal): 21

Q23_InfluencedToAnswerWithTheCompanyDesiredAction):

Gain Ratio feature evaluator

Ranked attributes:

0.25898 1 Q20_1_OpenEmailThatHas_ConvincingTitle

0.11872 12 Q21_9_DeterminePositiveAnswerToTheEmail_A_Catchy_Message

0.11744 16 Q21_13_DeterminePositiveAnswerToTheEmail_AClearMessage

0.11305 8 Q21_4_DeterminePositiveAnswerToTheEmail_ARepresentativePicture

0.09573 20 Q21_17_DeterminePositiveAnswerToTheEmail_ToBeConvincing

0.08826 15 Q21_12_DeterminePositiveAnswerToTheEmail_CompanyNameAppearance

0.08462 2 Q20_4_OpenEmailThatHas_TheCompanyName

0.07792 19 Q21_16_DeterminePositiveAnswerToTheEmail_DoNotBeIntrusive

0.07553 17 Q21_14_DeterminePositiveAnswerToTheEmail_AConciseMessage

0.069817 Q21_3_DeterminePositiveAnswerToTheEmail_ALinkForMoreDetailsToBeIncluded

0.06675 5 Q21_1_DeterminePositiveAnswerToTheEmail_ACompanyLogoToBeIncluded 


\begin{tabular}{|cc|}
\hline 0.06628 & 11 Q21_8_DeterminePositiveAnswerToTheEmail_RelevantInformation \\
0.06509 & 18 Q21_15_DeterminePositiveAnswerToTheEmail_ToIncludeAPromotionalOffer \\
0.05615 & 9 Q21_5_DeterminePositiveAnswerToTheEmail_AFunnyMessage \\
0.05566 & 13 Q21_10_DeterminePositiveAnswerToTheEmail_ACustomizedByeGreeting \\
0.05247 & 10 Q21_7_DeterminePositiveAnswerToTheEmail_ToHaveEnoughDetailsWithoutALink \\
0.04953 & 14 Q21_11_DeterminePositiveAnswerToTheEmail_TheSignatureAppearanceOf \\
0.04459 & 6 Q21_2_DeterminePositiveAnswerToTheEmail_ACustomizedGreeting \\
0.03888 & 3 Q20_5_OpenEmailThatHas_TheCompanyEmployerName \\
0.00209 & 4 Q20_6_OpenEmailThatHas_AnotherReason
\end{tabular}

According to the mentioned results, we can say that for the client, there is a strong correlation between the action of opening an email and the email subject $(0,25898)$, the message type $(0,11872)$, the message clearance $(0,11744)$ and the presence of a representative picture $(0,11305)$. Other attributes are ranked below 0,1 .

B. Clustering process - for achieving the clustering process results, the EM (Expectation Maximization) and "SimpleKMeans" clustering algorithms were used for grouping similar clients in groups. The EM algorithm was used to identify the approximated cluster numbers. In this case, the result was 3 clusters. This value was used as a parameter for the "SimpleKMeans" algorithm. The algorithm results are presented in Table 2.

Table 2.

Clients clusters based on influence factors related to open email activity

\begin{tabular}{|c|c|c|c|c|}
\hline No & Attribute & $\begin{array}{l}\text { Cluster 0 } \\
(105,40 \%)\end{array}$ & $\begin{array}{l}\text { Cluster1 } \\
(84,32 \%)\end{array}$ & $\begin{array}{l}\text { Cluster } 2 \\
(73,28 \%)\end{array}$ \\
\hline 1 & Q20_1_OpenEmailThatHas_ConvincingTitle & Yes & Indifferent & Yes \\
\hline 2 & Q20_4_OpenEmailThatHas_TheCompanyName & Yes & Indifferent & Indifferent \\
\hline 3 & $\begin{array}{l}\text { Q20_5_OpenEmailThatHas_TheCompanyEmployerNa } \\
\text { me }\end{array}$ & Indifferent & Indifferent & Indifferent \\
\hline 4 & Q20_6_OpenEmailThatHas_AnotherReason & $\begin{array}{l}\text { A_Catchy_ } \\
\text { Message }\end{array}$ & $\begin{array}{c}\text { A_Catchy_M } \\
\text { essage }\end{array}$ & $\begin{array}{l}\text { A_Catchy_ } \\
\text { Message }\end{array}$ \\
\hline 5 & $\begin{array}{l}\text { Q21_1_DeterminePositiveAnswerToTheEmail_AComp } \\
\text { anyLogoToBeIncluded }\end{array}$ & Yes & Indifferent & Indifferent \\
\hline 6 & $\begin{array}{l}\text { Q21_2_DeterminePositiveAnswerToTheEmail_ACusto } \\
\text { mizedGreeting }\end{array}$ & Yes & Indifferent & Indifferent \\
\hline 7 & $\begin{array}{l}\text { Q21_3_DeterminePositiveAnswerToTheEmail_ALinkF } \\
\text { orMoreDetailsToBeIncluded }\end{array}$ & Yes & Indifferent & Yes \\
\hline 8 & $\begin{array}{l}\text { Q21_4_DeterminePositiveAnswerToTheEmail_AR } \\
\text { epresentativePicture }\end{array}$ & Yes & Indifferent & Indifferent \\
\hline
\end{tabular}




\begin{tabular}{|c|c|c|c|c|}
\hline 9 & $\begin{array}{l}\text { Q21_5_DeterminePositiveAnswerToTheEmail_AFunny } \\
\text { Message }\end{array}$ & Indifferent & Indifferent & Indifferent \\
\hline 10 & $\begin{array}{l}\text { Q21_7_DeterminePositiveAnswerToTheEmail_ToHave } \\
\text { EnoughDetailsWithoutALink }\end{array}$ & Indifferent & Indifferent & Indifferent \\
\hline 11 & $\begin{array}{l}\text { Q21_8_DeterminePositiveAnswerToTheEmail_Relevan } \\
\text { tInformation }\end{array}$ & Yes & Indifferent & Indifferent \\
\hline 12 & $\begin{array}{l}\text { Q21_9_DeterminePositiveAnswerToTheEmail_A_Catc } \\
\text { hy_Message }\end{array}$ & Yes & Indifferent & Yes \\
\hline 13 & $\begin{array}{l}\text { Q21_10_DeterminePositiveAnswerToTheEmail_ACust } \\
\text { omizedByeGreeting }\end{array}$ & Indifferent & Indifferent & Indifferent \\
\hline 14 & $\begin{array}{l}\text { Q21_11_DeterminePositiveAnswerToTheEmail_ } \\
\text { TheSignatureAppearanceOfTheCompanyEmployer }\end{array}$ & Yes & Indifferent & Indifferent \\
\hline 15 & $\begin{array}{l}\text { Q21_12_DeterminePositiveAnswerToTheEmail_Compa } \\
\text { nyNameAppearance }\end{array}$ & Yes & Indifferent & Indifferent \\
\hline 16 & $\begin{array}{l}\text { Q21_13_DeterminePositiveAnswerToTheEmail_AClear } \\
\text { Message }\end{array}$ & $\begin{array}{l}\text { To_be } \\
\text { clear }\end{array}$ & Indifferent & $\begin{array}{l}\text { To_be_ } \\
\text { clear }\end{array}$ \\
\hline 17 & $\begin{array}{l}\text { Q21_14_DeterminePositiveAnswerToTheEmail_AConc } \\
\text { iseMessage }\end{array}$ & $\begin{array}{l}\text { To_be_ } \\
\text { concise }\end{array}$ & Indifferent & Indifferent \\
\hline 18 & $\begin{array}{l}\text { Q21_15_DeterminePositiveAnswerToTheEmail_ToIncl } \\
\text { udeAPromotionalOffer }\end{array}$ & Yes & Indifferent & Indifferent \\
\hline 19 & $\begin{array}{l}\text { Q21_16_DeterminePositiveAnswerToTheEmail_DoNot } \\
\text { BeIntrusive }\end{array}$ & Indifferent & Indifferent & Indifferent \\
\hline 20 & $\begin{array}{l}\text { Q21_17_DeterminePositiveAnswerToTheEmail_ToBeC } \\
\text { onvincing }\end{array}$ & $\begin{array}{c}\text { To_be_ } \\
\text { convincing }\end{array}$ & Indifferent & $\begin{array}{l}\text { To_be_ } \\
\text { convincing }\end{array}$ \\
\hline 21 & $\begin{array}{l}\text { Q23_InfluencedToAnswerWithTheCompanyDesiredAct } \\
\text { ion }\end{array}$ & Little & $\begin{array}{c}\text { Not_influenc } \\
\text { ed_at_all }\end{array}$ & Little \\
\hline
\end{tabular}

Taking into consideration the obtained results in Table 2, we can highlight the following aspects related to the three formed clusters (S1):

- Cluster 0: includes 105 instances from a total number of 262, representing $40 \%$ of the respondents. Clients from this cluster are characterized by the following aspects: they consider as being important the subject of the email, the appearance of the company name, the content of the message to be catchy, convincing, concise and clear. The email must also include the company logo, a promotional offer, relevant information, a customized offer, a link for more details and a representative picture. There are also some aspects that do not matter for these clients, for instance: a personalized bye greeting, the appearance of the name of the company representative. These clients might be influenced a little in doing an action desired by the organization in the email; 
- Cluster 1: includes 84 instances (32\% of the respondents). Users exposed to email marketing campaigns that belong to this cluster are indifferent to most of the aspects, but these are the ones that are not influenced at all to do any action that the company might want. They only consider important the message to be catchy;

- Cluster 2: includes 73 instances (28\% of the respondents). These persons might be influenced a little in doing the action desired by the organization and they consider as being important: the subject of the email, a link to be included in the email for more details, the message to be clear and catchy. The other aspects do not matter that much for these clients.

In figures 2 and 3 there are presented the clusters distribution from the point of view of the subject and the organization's name, based on the influence that they have over the client to open the email.

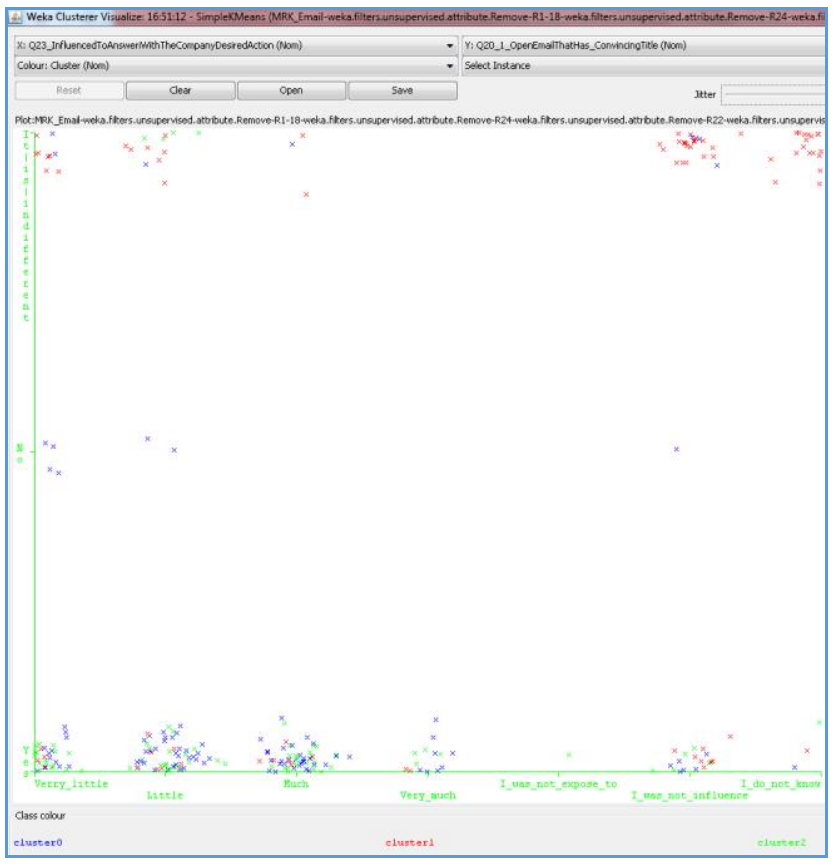

Fig. 2 Convincing email subject

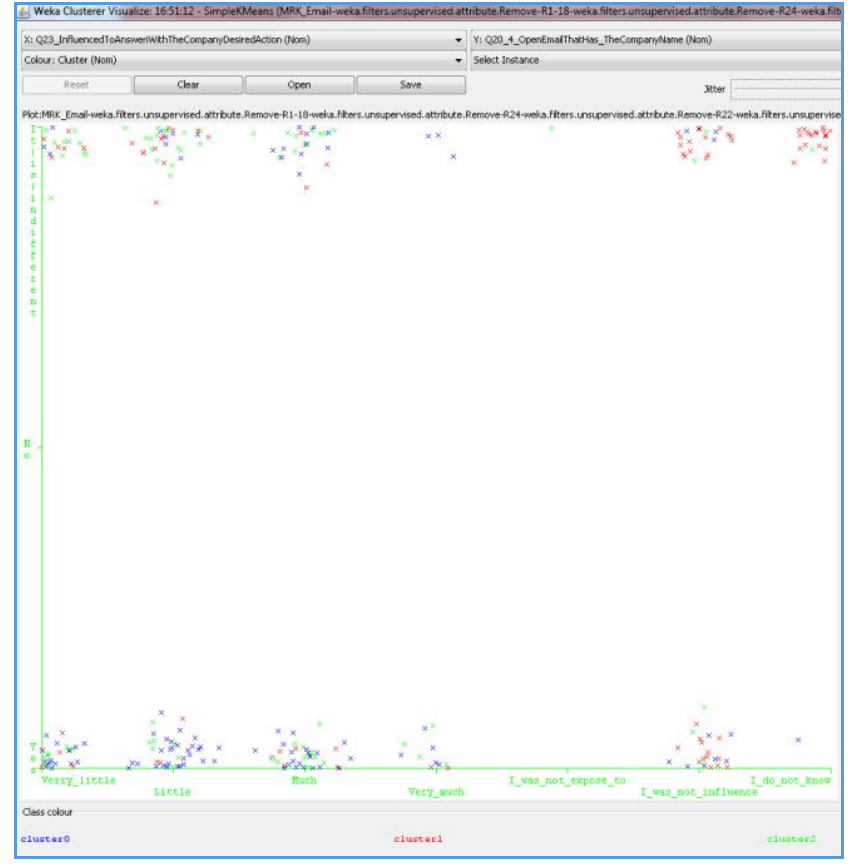

Fig. 3 Name of the organization

Figure 2 reveals the cluster assignment relation based on the influence over the client to take the expected action desired by the company in relation to the subject of the email. Clients from Cluster 0 (blue dots) and Cluster 2 (green dots) consider that the subject of the email is important. Clients from Cluster 1 (red dots) are indifferent to the subject the organization selected for the email.

Figure 3 reveals the cluster assignment relation based on the influence over the client to take the expected action desired by the company in relation to the appearance of the organization's name within the email. Clients from Cluster 0 (blue dots) consider that the company name is important to be included, whereas clients from Cluster 1 (red dots) and Cluster 2 (green dots) are indifferent to the inclusion of the company name.

C. Decision rule PART algorithm is used to identify clients' profiles that satisfied several conditions related to analyzed attributes. The class attribute is Q23 _InfluencedToAnswerWithTheCompanyDesiredAction. The algorithm builds a partial C4.5 decision tree in each iteration and makes the "best" leaf into a rule. Overall it was a set of 49 rules, but the most relevant rules are listed below (S2):

In order for the client to be influenced to answer to the email with the desired action wanted by the company, the email should have a convincing subject and a catchy message. Neither the logo of the company or the name of the person who signs the email, included in the body of the message does not influence the recipients to respond in that moment to that certain email (see Much_Rule 
below).

In order for the client to be influenced a little to answer to the email with the desired action wanted by the company, the email should have a convincing subject and should include a representative picture. Neither the included information, a link for more details and a promotional offer does not influence the recipients to better respond to that certain email.

A client that does not recognize if he was or not influenced to answer to the email with the desired action wanted by the company, considers as not important aspects like: email subject, company name appearance in email or the content of the email.

A client that considers that he was not at all influenced to answer to the email with the desired action wanted by the company is indifferent to aspects like email subject, message type (to be a catchy message) and the email to include a promotional offer.

Based on the information extract from the software, there were 197 correctly classified instances (75\%) and 65 incorrectly classified instances (25\%) and a Kappa statistic approximately 0.69 . The confusion matrix is:

a b c d e $\mathrm{f} g$ <-- classified as

\begin{tabular}{lllllll|l}
37 & 5 & 2 & 0 & 0 & 2 & 0 & $a=$ Very_little
\end{tabular}

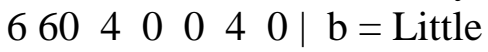

\begin{tabular}{lllllll|l}
5 & 5 & 48 & 1 & 0 & 3 & 0 & $\mathrm{c}=$ Much
\end{tabular}

\begin{tabular}{lllllll|l}
1 & 3 & 4 & 6 & 0 & 0 & 0 & $\mathrm{~d}=$ Very_much
\end{tabular}

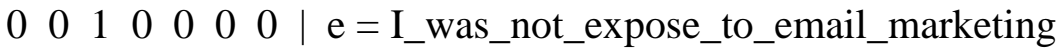

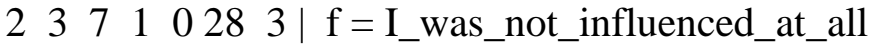

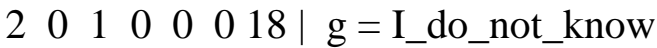

The confusion matrix indicates the number of the correct and incorrect classified instances. According to it, there are 37 clients correctly classified with Very_little (is about the factors influence over the recipients to respond in that moment to that certain email), 60 with Little, 48 with Much, 6 with Very_much, 28 with I_was_not_influenced_at_all and 18 with I_do_not_know.

Reading the first line means that 37 clients correctly classified with Very_little, 5 incorrectly classified with Little, 2 incorrectly classified with Much, 2 with I_was_not_influenced_at_all and 0 with Very_much, I_was_not_expose_to_email_marketing and I_do_not_know.

In order to identify the most important factors that influence the customers to buy a product / service based on a received email from a company (O2) it was selected the class attribute Q10_4_InfluencedToBuy_ByTheInformationReceivedByEmail.

A. Ranking attributes: this step was done in order to identify the relation between the class attribute and the others. Using "GainRatioAttributeEval" attribute evaluator and "Ranker" ordering method for selecting attributes, there were obtained the following results:

Attribute Evaluator (supervised, Class (nominal): 1

Q10_4_InfluencedToBuy_ByTheInformationReceivedByEmail):

Gain Ratio feature evaluator

Ranked attributes:

0.09362 2 Q20_1_OpenEmailThatHas_ConvincingTitle

0.0786317 Q21_13_DeterminePositiveAnswerToTheEmail_AClearMessage

0.0695310 Q21_5_DeterminePositiveAnswerToTheEmail_AFunnyMessage

0.05689 9 Q21_4_DeterminePositiveAnswerToTheEmail_ARepresentativePicture

0.04697 21 Q21_17_DeterminePositiveAnswerToTheEmail_ToBeConvincing

0.04337 18 Q21_14_DeterminePositiveAnswerToTheEmail_AConciseMessage

0.03978 13 Q21_9_DeterminePositiveAnswerToTheEmail_A_Catchy_Message

0.03816 20 Q21_16_DeterminePositiveAnswerToTheEmail_DoNotBeIntrusive 


\begin{tabular}{|l}
\hline 0.0377 19 Q21_15_DeterminePositiveAnswerToTheEmail_ToIncludeAPromotionalOffer \\
0.03479 3 Q20_4_OpenEmailThatHas_TheCompanyName \\
0.02796 12 Q21_8_DeterminePositiveAnswerToTheEmail_RelevantInformation \\
0.02538 16 Q21_12_DeterminePositiveAnswerToTheEmail_CompanyNameAppearance \\
0.02083 15 \\
Q21_11_DeterminePositiveAnswerToTheEmail_TheSignatureAppearanceOfCompanyEmployer \\
0.02057 4 Q20_5_OpenEmailThatHas_TheCompanyEmployerName \\
0.01973 7 Q21_2_DeterminePositiveAnswerToTheEmail_ACustomizedGreeting \\
0.01895 14 Q21_10_DeterminePositiveAnswerToTheEmail_ACustomizedByeGreeting \\
0.01488 8 Q21_3_DeterminePositiveAnswerToTheEmail_ALinkForMoreDetailsToBeIncluded \\
0.01011 6 Q21_1_DeterminePositiveAnswerToTheEmail_ACompanyLogoToBeIncluded \\
0.00916 5 Q20_6_OpenEmailThatHas_AnotherReason \\
0.00492 11 Q21_7_DeterminePositiveAnswerToTheEmail_ToHaveEnoughDetailsWithoutALink
\end{tabular}

According to the results, we can say that for the client, there is a strong correlation between the buying action and the email subject $(0,09362)$, the message clearance $(0,07863)$, the message type $(0,06953)$, and the presence of a representative picture $(0,05689)$. Other attributes are ranked below 0,05 . in Table 3:

B. Clustering process is similar with the one from previous section. The results are presented

Table 3.

Clients clusters based on influence factors related to influence in buying decision

\begin{tabular}{|c|c|c|c|c|}
\hline No & Attribute & $\begin{array}{l}\text { Cluster 0 } \\
(111,42 \%)\end{array}$ & $\begin{array}{l}\text { Cluster1 } \\
(76,29 \%)\end{array}$ & $\begin{array}{l}\text { Cluster } 2 \\
(75,29 \%)\end{array}$ \\
\hline 1 & Q20_1_OpenEmailThatHas_ConvincingTitle & Yes & Indifferent & Yes \\
\hline 2 & Q20_4_OpenEmailThatHas_TheCompanyName & Yes & Indifferent & Indifferent \\
\hline 3 & $\begin{array}{l}\text { Q20_5_OpenEmailThatHas_TheCompanyEmploy } \\
\text { erName }\end{array}$ & Indifferent & Indifferent & Indifferent \\
\hline 4 & Q20_6_OpenEmailThatHas_AnotherReason & $\begin{array}{l}\text { A_Catchy_ } \\
\text { Message }\end{array}$ & $\begin{array}{l}\text { A_Catchy_ } \\
\text { Message }\end{array}$ & $\begin{array}{l}\text { A_Catchy_ } \\
\text { Message }\end{array}$ \\
\hline 5 & $\begin{array}{l}\text { Q21_1_DeterminePositiveAnswerToTheEmail_A } \\
\text { CompanyLogoToBeIncluded }\end{array}$ & Yes & Indifferent & Indifferent \\
\hline 6 & $\begin{array}{l}\text { Q21_2_DeterminePositiveAnswerToTheEmail_A } \\
\text { CustomizedGreeting }\end{array}$ & Yes & Indifferent & Indifferent \\
\hline 7 & $\begin{array}{l}\text { Q21_3_DeterminePositiveAnswerToTheEmail_A } \\
\text { LinkForMoreDetailsToBeIncluded }\end{array}$ & Yes & Indifferent & Yes \\
\hline 8 & Q21_4_DeterminePositiveAnswerToTheEmail_A & Yes & Indifferent & Indifferent \\
\hline
\end{tabular}




\begin{tabular}{|c|c|c|c|c|}
\hline & RepresentativePicture & & & \\
\hline 9 & $\begin{array}{l}\text { Q21_5_DeterminePositiveAnswerToTheEmail_A } \\
\text { FunnyMessage }\end{array}$ & Indifferent & Indifferent & Indifferent \\
\hline 10 & $\begin{array}{l}\text { Q21_7_DeterminePositiveAnswerToTheEmail_T } \\
\text { oHaveEnoughDetailsWithoutALink }\end{array}$ & Indifferent & Indifferent & Indifferent \\
\hline 11 & $\begin{array}{l}\text { Q21_8_DeterminePositiveAnswerToTheEmail_R } \\
\text { elevantInformation }\end{array}$ & Yes & Indifferent & Indifferent \\
\hline 12 & $\begin{array}{l}\text { Q21_9_DeterminePositiveAnswerToTheEmail_A } \\
\text { _Catchy_Message }\end{array}$ & Yes & Indifferent & Yes \\
\hline 13 & $\begin{array}{l}\text { Q21_10_DeterminePositiveAnswerToTheEmail_ } \\
\text { ACustomizedByeGreeting }\end{array}$ & Indifferent & Indifferent & Indifferent \\
\hline 14 & $\begin{array}{l}\text { Q21_11_DeterminePositiveAnswerToTheEmail_ } \\
\text { TheSignatureAppearanceOfTheCompanyEmploye } \\
\text { r }\end{array}$ & Yes & Indifferent & Indifferent \\
\hline 15 & $\begin{array}{l}\text { Q21_12_DeterminePositiveAnswerToTheEmail_ } \\
\text { CompanyNameAppearance }\end{array}$ & Yes & Indifferent & Indifferent \\
\hline 16 & $\begin{array}{l}\text { Q21_13_DeterminePositiveAnswerToTheEmail_ } \\
\text { AClearMessage }\end{array}$ & $\begin{array}{l}\text { To_be_ } \\
\text { clear }\end{array}$ & Indifferent & $\begin{array}{l}\text { To_be_ } \\
\text { clear }\end{array}$ \\
\hline 17 & $\begin{array}{l}\text { Q21_14_DeterminePositiveAnswerToTheEmail_ } \\
\text { AConciseMessage }\end{array}$ & $\begin{array}{l}\text { To_be_- } \\
\text { concise }\end{array}$ & Indifferent & Indifferent \\
\hline 18 & $\begin{array}{l}\text { Q21_15_DeterminePositiveAnswerToTheEmail_ } \\
\text { ToIncludeAPromotionalOffer }\end{array}$ & Yes & Indifferent & Indifferent \\
\hline 19 & $\begin{array}{l}\text { Q21_16_DeterminePositiveAnswerToTheEmail_ } \\
\text { DoNotBeIntrusive }\end{array}$ & Indifferent & Indifferent & Indifferent \\
\hline 20 & $\begin{array}{l}\text { Q21_17_DeterminePositiveAnswerToTheEmail_ } \\
\text { ToBeConvincing }\end{array}$ & $\begin{array}{l}\text { To_be_ } \\
\text { convincing }\end{array}$ & Indifferent & $\begin{array}{l}\text { To_be_ } \\
\text { convincing }\end{array}$ \\
\hline 21 & $\begin{array}{l}\text { Q10_4_InfluencedToBuy_ByTheInformationRece } \\
\text { ivedByEmail }\end{array}$ & Little & Very_much & Much \\
\hline
\end{tabular}

The results are showing the following information about the clusters (S1):

- for clients from Cluster 0 (111 instances that represent $42 \%$ ) the factors have little influence regarding the buying action, despite the fact that the clients are taking into account these aspects. These aspects are: email subject, company name, to be a catchy message, a company logo to be included, a personalized greeting, a link for finding out more details, a 
representative picture, relevant information, and the appearance of the signature of the company representative, a clear and concise message, to include a promotional offer;

- clients from Cluster 1 (76 instances, 29\%) are influenced very much by these factors even if they appear to be indifferent to the them;

- clients from Cluster 2 (75 instances, 29\%)are also indifferent to these factors except those related to the subject, to the hyperlink for more details, the message clearance. Based on this conclusion, the factors have a big influence over the clients to buy the promoted product / service.

In figures 4 and 5 are depicted the influencing factors like email title and message clearance over the buying action:

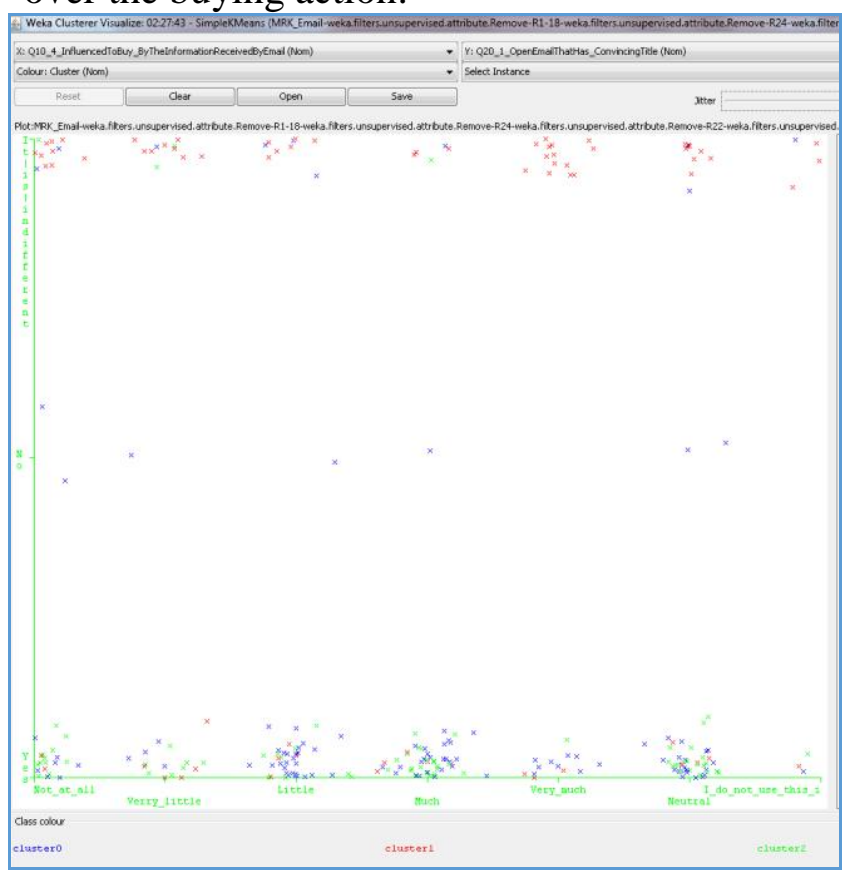

Fig. 4 Convincing email subject

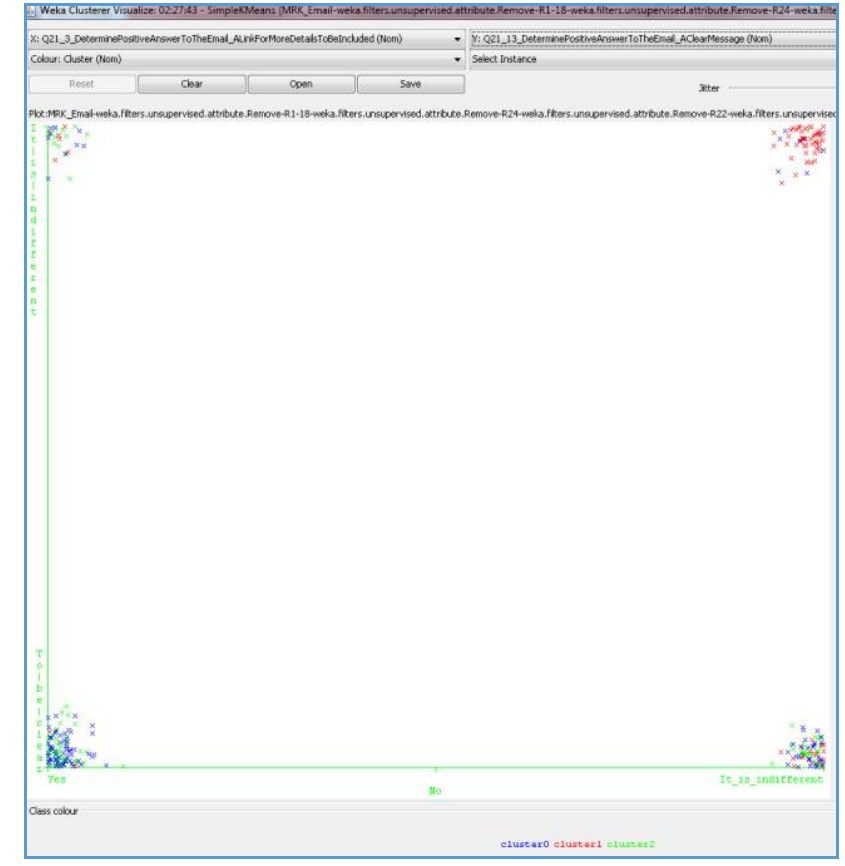

Fig. 5 A clear message

Figure 4 reveals the cluster assignment relation based on the influence to buy (based on the information received by email) in relation to the email's subject. Clients from Cluster 0 (blue dots) and Cluster 2 (green dots) consider that a convincing email subject is important, whereas clients from Cluster 1 (red dots) are indifferent to the subject selected for the email.

Figure 5 reveals the cluster assignment relation based on the influence to buy (based on the information received by email) in relation to the message clearance. Clients from Cluster 0 (blue dots) and Cluster 2 (green dots) consider that is important for an email to contain a clear message. Clients from Cluster 1 (red dots) are indifferent to this aspect.

C. Decision rule PART algorithm is used to identify clients' profiles that satisfied several conditions related to analyzed attributes. The class attribute is Q10_4_InfluencedToBuy_ByTheInformationReceivedByEmail. The algorithm builds a partial C4.5 decision tree in each iteration and makes the "best" leaf into a rule. Overall it was a set of 23 rules, but the most relevant rules are listed below (S2):

The clients would be much influenced to buy if the email received has a convincing subject, a catchy message, it includes a representative picture of the promoted product / service and it is included the name of the person who signs the email on behalf of the company. The name of the employee seems to be determinant, since without its inclusion, the clients would only be influenced a little to make a buying. The decision rule for this situation is mentioned below, having 14 clients that respect this condition:

Q20_6_OpenEmailThatHas_AnotherReason = A_Catchy_Message AND 
Q20_1_OpenEmailThatHas_ConvincingTitle $=$ Yes AND

Q21_4_DeterminePositiveAnswerToTheEmail_ARepresentativePicture $=$ Yes AND

Q20_5_OpenEmailThatHas_TheCompanyEmployerName = Yes: Much (14/0)

The clients would be also much influenced to buy if the email received has a catchy, convincing and clear message, a convincing subject and comprises relevant information for the person's current needs. A representative picture included in the email seems to be not so important to the clients.

The clients would have a neutral attitude in what concerns the buying decision if the email received has a catchy message. A representative picture included in email, a personalized ending greeting and enough information in the email without being necessary a link seems to be irrelevant to these clients. decision.

Having a short subject of the email would not influence in a great manner the buying

Based on the information extract from the software, there was 102 correctly classified instances (39\%) and 160 incorrectly classified instances (61\%) and a Kappa statistic approximately 0.23 . The confusion matrix is:
a b c d e $\mathrm{f} g$ <-- classified as

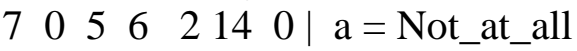
\begin{tabular}{lllllll|l}
3 & 2 & 0 & 8 & 4 & 11 & 0 & $b=$ Very_little
\end{tabular}
\begin{tabular}{lllllll|l}
4 & 1 & 9 & 14 & 1 & 20 & 0 & $\mathrm{c}=$ Little
\end{tabular}
\begin{tabular}{lllllll|l}
1 & 0 & 2 & 31 & 0 & 25 & 0 & $\mathrm{~d}=$ Much
\end{tabular}
\begin{tabular}{lllllll|l}
1 & 0 & 1 & 7 & 13 & 5 & 0 & e $=$ Very_much
\end{tabular}
\begin{tabular}{lllllll|l}
1 & 0 & 5 & 12 & 3 & 37 & 0 & $\mathrm{f}=$ Neutral
\end{tabular}
\begin{tabular}{lllllll|l}
0 & 0 & 0 & 0 & 0 & 4 & 3 & $\mathrm{~g}=$ I_do_not_use_this_information_source
\end{tabular}

As we can see, the number of incorrectly classified instances is big. Also the Kappa statistic has a value that indicates a poor correlation that exists in data from this point of view. So, a set of good conclusion cannot be highlighted for this research direction. Still, for the clustering process, the results may be a good starting point for creating pattern email. Probably this number is influenced by the fact that the majority of the respondents are aged 20-22 and they may not be the ones making the buying decisions.

The research conducted has provided several conclusions regarding the way email marketing campaigns should be designed in order to better satisfy the consumers:

- aspects such as: the subject of the email, presence of a representative picture, information about the company / representative are important to some clients. These aspects may influence to some extent the client's decision to take an action desired by the company when an email is received and opened, and also to buy the promoted product / service. The company that designs email marketing campaigns should pay attention to the aspects mentioned. They will make a difference if the client acts, as the company wants, or not. This client category is very confident in the information comprised in the emails received;

- there are also some client categories that will pay attention only to aspects: like email subject, company logo and name to be included in the email and the message to be catchy. In this situation, the company must take into account these aspects more than the others. This client category is a little bit informed about the product / service wanted to be bought;

- another client category is the one that is indifferent to the email marketing campaigns. Aspects like the ones that were analyzed are not relevant to these clients because they already know what to buy and from whom. They search for information about the product / service, knowing enough details about them. 


\section{CONCLUSIONS, LIMITS OF THE RESEARCH AND FURTHER RESEARCH DIRECTIONS}

Based on the findings from this research, the authors draw attention to organizations upon the importance of the subject of the email. This should be a phrase that encompasses a call-to-action so that it would convince the recipient to open the email. The subject of the email should be related to the content of the email, it should be compelling and it should make the reader curious and excited to open and find out what the email is about. It is not recommended to be too long, to be properly displayed on mobile devices, 5-7 words should be convincing enough.

The authors recommend the email to start with a personalized greeting, either with 'Dear $\mathrm{Mr} / \mathrm{s}$. FamilyName' or with 'Hi/Hello FirstName' of the recipient since it creates a more personal approach - this greeting formula should be adapted to the characteristics of the selected target and to the products / services offered.

The first paragraph of the email should contain the most important information and it is meant to grab the attention of the reader. The message should be written so that it highlights the benefits that the recipient has if he makes the action wanted by the company (book a ticket to an event promoted, find out more information about the promoted product / service, attend a webinar etc.). In the content of the message it should be included a representative picture (maximum two photographs - one can be the company's logo) of the product / service / event that is promoted and their title / name (alt text that appears if the recipient does not display the images of the email), also a hyperlink (maximum two links) to a landing page that contains more information (for those persons interested to find out more, or to order). At least one of the links included should have the form: www.website.domain/something_representative so that any reader observes the existence of a hyperlink in the text and is attracted to click on it. The other link (that should lead to the same landing page) may have the form of a button that has a call-to-action text on it, and a color that impulses the target to click on it (for instance: green, blue, orange - red is not advised to be used since it sends a 'stop' message, unless the company's corporate image / logo includes that color). Organizations should not be tempted to write long and colorful emails, a sufficient length of a direct email is about 100 words and keywords should be highlighted by using bold or italic words (not larger fonts, not many colors, maximum two colors).

The ending salutation should be in accordance with the personalized greeting (formal or informal) and it should include (on separate rows): the First and Last name of the representative of the company (on behalf of whom the email is sent and who can offer additional information if contacted by the recipient of the email); his / her position in the company; the name of the company (if it had not already been mentioned in the content of the message); and contact information (telephone number and email address). It is not advised to sign the paper on behalf of the company or from a department because it has lower open rates (Shah, 2013).

It is a good idea to include social-sharing buttons, so that the recipient can share the content of the email with a friend (make a recommendation), post it on the social networks he / she uses: Facebook, Twitter, Google+, LinkedIn etc and / or social-following buttons, so that the recipient can become a fan of your Facebook page, can access directly your website or contact you by email. The recommendation would be to place the social-sharing buttons in the top of the email and the social-following buttons at the end of the email.

At the bottom of the email the section Disclaimer is compulsory in order to respect the law that constraints organizations to give the recipient of an email, the possibility to unsubscribe from the database. The name of the organization and its business address are mandatory to appear in the emails designed.

Relevance of the research consists in the fact that identifying customer expectations and creating customer groups with similar expectations, pattern emails can be created. These emails are very likely to have a larger impact on the client. Based on the client profile, factors such as the email subject, content, greeting formula, and information within the email are very important. There 
are also other relevant issues related to the company that sends the email. Once created these patterns of emails, the company that wants to promote a product / service may reuse them depending on which group a client is assigned.

The major limitation of the research consists in the low number of respondents (only 262), so this exploratory research should be followed by a conclusive one to verify the conclusions of the present research. Also, the majority of the respondents were aged 20-22 and this might have influenced the results obtained for $\mathrm{O} 2$ (maybe because they are not the ones who take the buying decision of goods or services). In what concerns data mining, the authors only used clustering and classification, but for a better data analysis, also some other algorithms based on neural networks (like Backpropagation) may be useful.

Future directions of research may include: adding some other information about respondents like the job, income, civil status, time spent in front of the computer, number of times email is accessed daily / weekly, the most used devices for connecting to the internet, number of articles bought online in a specific period of time. Present paper only researched the stage of content developing, but future direction of research could include the other stages of the email marketing process.

\section{ACKNOWLEDGEMENT}

This work was cofinanced from the European Social Fund through Sectoral Operational

Programme Human Resources Development 2007-2013, project number POSDRU/159/1.5/S/134197 „Performance and excellence in doctoral and postdoctoral research in Romanian economics science domain"

\section{REFERENCES}

1. Angeles, S., 2014. 11 Email Marketing Solutions for Small Businesses, available: http://www.businessnewsdaily.com/5829-email-marketing-solutions.html, accessed March 2015.

2. Blyth, A., 2011. Brilliant online marketing. How to use internet to market your business, Edinburgh, Pearson. Ch. 6 Email marketing.

3. Chaffey, D., Smith, P.R., 2008. eMarketing eXcellence - Planning and optimizing your digital marketing, Burlington, Butterworth-Heinemann.

4. Chapman, P., Clinton, J., Kerber, R., Khabaza, T., Reinartz, T., Shearer, C., Wirth, R., 2000. CRISP-DM 1.0 Step-by-step data mining guide, The CRISPDM Consortium / SPSS Inc. Available http://www.iidia.com.ar/rgm/CD-TIpEI/TEI-2-CRISP-DM-GdP-material.pdf, accessed May 2015.

5. Charlesworth, A., 2009. Internet Marketing - A practical approach, Burlington, Butterworth-Heinemann. Ch. 8 Permission marketing.

6. Cioană, G., Dinulescu, R., 2015. Improving Public Health Insurance Experience for Patients and Family Doctors' Work Effectiveness through Lean Six Sigma Tools: the National Health Insurance Card - Evidence from Romania, Proceedings of The 25th International Business Information Management Association Conference "Innovation Vision 2020: From Regional Development Sustainability to Global Economic Growth " May 7-8, 2015, Amsterdam, Netherlands, pp. 3089-3098, ISBN: 9780986041945.

7. Duistermaat, H., 2013. 37 Tips for Writing Emails that Get Opened, Read, and Clicked, Available: http://www.copyblogger.com/37-email-marketing-tips/, accessed April 2015.

8. Fallon, N., 2014. 4 Email Marketing Tricks That Work, Available: http://www.businessnewsdaily.com/5999-effective-email-marketing-tips.html, accessed March 2015.

9. Ford, J., 2014. 8 Email Marketing Tips and Tricks, Available: 
http://quickbooks.intuit.com/r/email-marketing/8-email-marketing-tips-tricks, March 2015.

10. Friedlein, A., 2003. Maintaining and Evolving Successful Commercial Web Sites. Managing Change, Content, Customer Relationships, and Site Measurement, San Francisco, Elsevier. Ch. 10 Personalization.

11. Gay, R., Charlesworth, A., Essen, R., 2007. Online marketing - A customer - led approach, Oxford: Oxford University Press, Ch. 11 Online communication tools.

12. Grimes, G.A., Hough, M.G., Signorella, M.L., 2007. Email end user and spam: relations of gender and age group to attitudes and actions, Computers in Human Behavior, Vol. 23, pp. 318-332.

13. Handley, A., 2012. How to Create an E-Mail Marketing Campaign That People Will Notice, Available: http://www.entrepreneur.com/article/224443, accessed April 2015.

14. Hurduzeu, R.E., 2015. The impact of leadership on organizational performance, SEA, Practical Application of Science, vol. III, 1(7), pp. 289-294.

15. Montgomery, A.L., Smith, M.D., 2009, Prospects for Personalization on the Internet, Journal of Interactive Marketing, Vol. 23, pp. 130-137.

16. Pantea, C., Pop, N.Al., 2010. Email marketing campaigns: the easiest path from organizations to consumers - an exploratory assessment, The Annals of the University of Oradea, Economic Sciences Fascicle - TOM XIX, pp. 737-742, ISSN 1582-5450.

17. Shah, N., 2013. 8 Little Tricks to Make Your Emails More Clickable, Available: http://blog.hubspot.com/marketing/make-emails-more-clickable-list, accessed May 2015.

18. Usadata.com, 7 Smart Email Marketing Tips, Available: http://www.usadata.com/seventips-emailmarketing.html, accessed March 2015.

19. Vegheș, C., 2003. Marketing direct, Bucharest, Uranus.

20. Waikato, 2015. Weka 3: Data mining software in Java, Available: http://www.cs.waikato.ac.nz/ml/weka/, accessed June 2015.

Yola, 2013. Marketing Campaigns 101: 7 Steps to Launching Your First Email Marketing Campaign, Available: https://www.yola.com/blog/marketing-campaigns-101-7-steps-to-launchingyour-first-email-marketing-campaign/, 